\title{
Energy-Effective Communication Based on Compressed Sensing
}

\author{
Wang Yin-yin \\ The First People's Hospital of Yancheng, Yancheng, P. R. China
}

Email address:

hobbyc@163.com

To cite this article:

Wang Yin-yin. Energy-Effective Communication Based on Compressed Sensing. American Journal of Networks and Communications. Vol. 5, No. 6, 2016, pp. 121-127. doi: 10.11648/j.ajnc.20160506.11

Received: November 5, 2016; Accepted: November 22, 2016; Published: December 8, 2016

\begin{abstract}
In order to improve energy-effect, Compressed Sensing has been employed gradually in the process of collaborative communication. For practical applications, localized features of local area are further considered in this technology, which is called classification CS. However, collaborative methods in current literatures are not so suitable in this scene that the advantages of CS could not be benefit. In this paper, a novel collaborative communication mechanism based on classification CS is proposed for actual environments. An effective collaborative transmission mode based on classification is presented, in which energy cost reduce effectively in the process of transmission and the reconstructed signals could reach at least the theoretical low bound to avoid redundant samplings. In experiments, our mechanism has been proved valuable and feasible in realistic applications.
\end{abstract}

Keywords: Collaborative Routing, Compressed Sensing, Communication, WSN

\section{Introduction}

In the process of transmitting information in Wireless Sensor Networks (WSNs), improving energy-effect of sensors is an especially important problem to prolong the lifetime of networks. Compressive Sensing (CS), as a novel and effective signal transforming technology, has been proposed in different fields, such as medical [1] and image [2]. At the same time, this technology has also been adopted gradually in WSN [3-11].

Compressive sensing is a signal sampling technique that acquires few sparse or compressible signals and then recovers them precisely. The transmitter only needs to send the sampled signals, which are obtained through a simple method of measurement, to the termination instead of compressing and encoding the original signals. After receiving the sampled signals, the termination can recover the original signals accurately through an effective recovery algorithm. Obviously, it is the significant advantage of CS technology that energy cost is rarely needed in the process of sampling signal. In this case, compressive sensing could be applied to reduce efficiently the energy cost of sensors in the process of collaborative communication. For instance, [4] proposes the idea of oversample for the robustness of data transmission. It obviously increases the energy cost of sensors and the risk of unnecessary sampling. [5-7] use CS technologies in some special scenes in order to construct sparsity signals. The effectiveness of the algorithms greatly depends on special scenarios in actual applications, as the original signals must be recognized as the sparse signals. The restrictive conditions confine the applicational space of these methods. [8-9] implies that the energy of the transmitting end was enough, which could not be applied in WSN on account of limited energy of sensors. For better collaborative communication, some researches based on localized characters in practice have been presented recently, such as [10] shown spatial-temporal correlation and [11] discussed the features of local region. These schemes are applied in different situations, however, they indicates a common idea that is classification based a certain standard could further improve the effects of CS.

Unfortunately, their transmission modes in these literatures are so simple and direct without any cooperation strategy, thus the processes of transportation are not energy-effective apparently based on classification. Compared with non-CS methods, it is known that bottom and side sensors in the network cost more energy in traditional CS. Hence, it would result in unnecessary energy waste considerably in practice, 
especially when the data are conveyed in the case of classification. Furthermore, it is difficult for traditional CS to know whether samplings are enough for accurate reconstruction. In other words, the receiving terminal could not realize when the process of signals sampling should be stopped in order to avoid redundant samplings.

In this paper, a novel collaborative communication mechanism based on classification CS is proposed to address these problems. For collaborative transmission, an effective transmission mode is proposed to reduce energy cost and a sampling principle is presented to avoid redundant samplings. In this work, we propose a hybrid scheme that both CS and non-CS are adopted for energy-effective transmissions. Based on the classified network, the transfer modes in both inter-classes and intra-classes are different. In inter-classes, we choose suitable relay nodes in a class which the closest to the sink in this class. Sensors directly convey original signals to their relays without using CS, since the number of hops is probably very small in a certain class. Therefore, these sensors may merely transmit data few times instead of the number which is equal to the required measurement times in the CS method. Subsequently, localized schemes mentioned above could be applied after these relay nodes receive all original signals of the remaining sensors of classes, which is very beneficial to reduce energy cost. That is, the functions of relay nodes in this transmission mode are not only a signal forwarder but also a processor. Afterwards, new values are produced and sent to the sink using CS in intra-classes. In this process, two conditions should be considered. One is that the scheme of sampling has to meet the requirement of irrelevance between measurement matrix and sparse matrix. The other is that a high accurate reconstruction is required to prevent error diffusion since the received data by the sink are not original signals. For the former, we present a "good" measurement matrix which is as effective as random matrix. For the later, we present a principle of effective sampling. It can reach the theoretical low bound of the accuracy of signal reconstruction. That is, invalid samplings can be avoided. Using our transport method, energy consumption of bottom and side sensors effectively decrease, while no extra transmissions are required for the rest sensors. Our experiments show that the energy cost in our method saves about at least $20 \%$. In particular, it saves about $60 \%$ when the number of sensors is not very large (say, no more than 50 ).

Our contributions in the paper are summarized as follows:

a) We propose a solution that both $\mathrm{CS}$ and non-CS are employed in the process of collaborative communication, which effectively reduces energy consumption of sensors.

b) The proved sampling principle could at least reach the theoretical low bound of the accuracy of the signal reconstruction, which avoids invalid samplings.

The rest of this paper is organized as follows. Section 2 introduces background of CS and DCS and the simple concepts of Ricci flow. Section 3 outlines the collaborative transmission mechanism which includes transmission. Discussion is presented in Section 4. Experiments in actual environments and simulations are in Section 5. Finally, conclusion is given in Section 6.

\section{Background and Relative Concepts}

In this section, we shall briefly review the natural compressive sensing theory, including Restricted Isometry Property and $l_{1}$ minimization reconstruction algorithm, which become the basis of CS technology. Furthermore, the theory of Distributed Compressed Sensing would be introduced. This theory explores mainly the internal character of signals and their mutual interrelations.

\subsection{Compressive Sensing Theory}

Compressive sensing [14-16] is a novel fusion technology in signal acquisition and compression reconstructing sparse signals in form of few random measurements. According to the theory of CS, it simultaneously samples and compresses signals which could have a sparse representation in some known basis or dictionaries in the process of sampling and recovers the original signal stably and exactly by linear programming.

Consider $\mathrm{C}$ is a $\mathrm{N}$-dimensional signal and $\Phi$ is $\mathrm{M} \times \mathrm{N}$ matrix, where $\mathrm{M}<\mathrm{N}$, and set $\mathrm{Y}=\Phi \times \mathrm{C}$, then $\mathrm{Y}$ is an $\mathrm{M}$ dimensional vector and could be considered as $M$ measurements from $\mathrm{C}$ through the measurement matrix $\Phi$. Based on the conclusion of CS theory, it is probable that the original signal $\mathrm{C}$ could be reconstructed precisely from the compressed signal $\mathrm{Y}$ if $\mathrm{C}$ can be represented as a linear combination of only $\mathrm{K}$ basis vectors, where $\mathrm{K}<\mathrm{M}$. Meanwhile, the measure matrix $\Phi$ must satisfy Restricted Isometry Property (RIP) in order to recover C stably. RIP could be expressed as: Let $\theta \in(0,1)$, for any $m \in[1, \min (N, M)]$, the restricted isometry constant of $\mathrm{C}$ is equal to the smallest quantity $\theta_{m}=\theta_{m}(\varphi)$ such that

$$
\left(1-\theta_{m}\right)\|C\|^{2} \leq\left\|\varphi_{I} C\right\|^{2} \leq\left(1+\theta_{m}\right)\|C\|^{2}
$$

for any subset I of $\varphi$ with $|I| \leq m$ and coefficient sequences $\left(C_{j}\right), j \in I$. The measure matrix $\Phi$ is called to satisfy the RIP of order $\mathrm{m}$ with parameter $\theta, 0 \leq \theta_{m}<\theta$. If equation (1) can be satisfied, then the signal $\mathrm{C}$ could be recovered accurately with high probability by solving the convex problem (2) or (3)

$$
\begin{gathered}
\operatorname{minimize}\|C\| l_{1} \text { subject to } \mathrm{Y}=\varphi \times \mathrm{C} \\
\text { minimize }\|C\| l_{1} \text { subject to }\|\varphi \times \mathrm{C}-\mathrm{Y}\| l_{1} \leq \lambda
\end{gathered}
$$

where the $l_{1}$-norm is defined by $\|C\| l_{1}=\sum_{i}\left|C_{i}\right|_{1}^{N}$ for each $C=C_{i} \in R^{N}, i \in[1, N]$ and $\lambda$ is an arbitrarily small constant. That is the so-called $l_{1}$ minimization reconstruction algorithm in CS technique.

Obviously, there are three aspects that need to be taken into account in the CS theory. First, a special sparse matrix 
consisted of several sparse basis is needed, which is able to transform the original signal $\mathrm{C}$ to a sparse signal. Second, a measurement matrix $\Phi\left(\Phi \in \mathrm{R}^{M \times N}\right.$ and $\left.\mathrm{M}<<\mathrm{N}\right)$ should be irrelevant to the sparse matrix. It is important to design an optimized measurement matrix to increase the accuracy of CS reconstruction. Finally, a reliable recovery algorithm should be adopted to reconstruct original signals.

\subsection{Distributed Compressive Sensing}

Distributed Compressed Sensing (DCS) has been proposed in [17] to exploit both intra- and inter-signal correlation structures to distributed code for mutli-signal ensembles. This theory rests on the concept that is called Joint Sparsity Model (JSM). Similar with the description of CS theory, consider a signal $\mathrm{X}$ is $\mathrm{N}$ dimensions vector $\left\langle x_{1}, x_{2}, \ldots . . x_{N}\right\rangle$, where $\left(x_{j}\right)_{j=1}^{N} \in R^{N}$. Let a sparse matrix $\Psi$ consisted of a set of sparse basis $\Psi_{\mathrm{i}}, 1 \leqslant \mathrm{i} \leqslant \mathrm{N}$, and each element $x_{j}$ can be represented sparsely by a subset of matrix $\Psi$.

Based on JSM, the signal $\mathrm{X}$ is generated as a combination of two parts, which are called the common component and the innovation component. In this case, all elements of the signal contain the same common part while each individual has a different innovation component. That is,

$$
x_{j}=z_{c}+z_{j}, \mathrm{j} \in(1,2, \ldots, \mathrm{N})
$$

where $z_{c}$ and $z_{j}$ denote the common component and the innovation component, respectively.

Different sparsity assumptions lead to various joint sparse models related to both the common part and the innovation part. The JSM-1 model is suitable for the actual applications in WSNs, which is given by:

$$
\begin{gathered}
x_{j}=z_{c}+z_{j}, \mathrm{j} \in(1,2, \ldots, \mathrm{N}) \\
\text { subject to } z_{c}=\psi \theta_{c} \\
\left\|\theta_{c} z\right\|_{0}=k_{c} \\
z_{j}=\psi \theta_{j} \\
\left\|\theta_{j} z\right\|_{0}=k_{j}
\end{gathered}
$$

In (5), the common component $z_{c}$ is $k_{c}$ sparse in the basis $\Psi$ and shared by each $\left(x_{j}\right)_{j=1}^{N} \in R^{N}$, and the innovation component $z_{j}$ is $k_{j}$ sparse in the same basis. \|\|$_{0}$ denotes the 0 -norm.

Under this model, the common part of the signal should be extracted as many as possible in order to obtain the recovered result precisely. In other words, the error rate of reconstruction will be decreased when the proportion of the abstracted common component is far more than the individual one in the signal, which is the reason of classification. In each class, the common component is abstract, such as [11], and the accuracy of reconstruction will be increased.

\section{Collaborative Transmission Based on CS}

In this section we will describe the collaborative transmission mode based on the mixed method. In the interclass, sensors transmit the gathering data to their relay nodes by the traditional transmission. After producing new data, the relay nodes convey them to the sink by CS. To reduce transmission, a sampling principle is presented and proved.

\subsection{In Inter-Class}

As we all know, an obvious advantage of CS technique is that energy cost of top sensors which are closer to the sink is the same as the one of bottom sensors, which is valuable for the load balancing of the whole network. However, this theoretical superiority is probably not embodied in actual environments, even worse. Compared with non-CS methods, bottom sensors in the network cost more energy in traditional $\mathrm{CS}$, though load balancing of the network has been reached. Hence, it would result in unnecessary energy waste considerably in practice. Consider $\mathrm{N}$ sensors are deployed in a localized region with one sink. That is, there are $\mathrm{N}$ sensors in the network and one of them is the sink sensor. If the number of measurement is $\mathrm{M}$, then $\mathrm{N}-1$ sensors must transmit their data packages $\mathrm{M}$ times according to $\mathrm{CS}$ theory. On the contrary, the number of necessary transmission without CS only depends on the number of their hops to the sink. In practice, this number is much less than $M$ since the region is localized. Sometimes, the required amount of transmission using CS is obvious more than the one without CS in real situations. An example is demonstrated in Section V.

Hence, we propose a simple yet effective transmission mode in inter-classes. A relay node $r$ in a class is selected to which the rest sensors send their data based on the given routes. That is, these sensors just need to transmit the same times as traditional transport modes. After receiving data packages, $r$ does not forwards these values directly but produces a new data $d_{\text {new }}$ using mentioned above schemes.

It is clear that the number of transmission will decrease and energy cost will reduce, since 1) the number of sensors in a class is probably less than the number of measurement owning to environment influences; 2) further features in actual environments could be applied after relay nodes receive all signals of the rest sensors. In this case, the number of transmission in inter-class is $\sum_{r \in R}(|S(r)|-1)$, where $R$ is a set of relay nodes and $|\bullet|$ denotes cardinality, $S(r)$ denotes a class whose relay node is $r$.

\subsection{In Intra-Class}

As for transmission mode in intra-classes, relay nodes only need to transport their new value $d_{\text {new }}$ s to the sink by CS technique. Nevertheless, there are two aspects which have to 
be noticed. One is that the incoherent between measurement matrix and sparse matrix should be satisfied based on the theory of CS. The other is that the accuracy of reconstruction is required of a high standard. For better effect of compression, some schemes are used in the process of producing new values and thus data which the sink receives are not original signals. In other words, these values are just "intermediate data". To get original signals accurately, intermediate data must be recovery very precisely to prevent error diffusion. Intuitively, the number of measurement should be large enough to recover signals high accurately [22]. Unfortunately, it is obvious that unnecessary transmissions would arise.

In this paper, a "good" measurement matrix and a sampling principle are presented for energy-effective transmission. The former is used to guarantee incoherence between measurement matrices and transform matrices, and the later is employed to decide whether the number of measurement is enough to reconstruct signals exactly.

Firstly, the set $\{-1,0,1\}$ is used to produce measurement matrices by probability selecting. The selection probabilities are $1 / 4,1 / 2,1 / 4$ respectively. Therefore, the occurrence probability of zero in a measurement matrix is equal to one of non-zero in theory. In this case, our measurement matrix will be effective as well as random matrices [7], which are excellent candidates for incoherence between measurement matrices and transform matrices [1].

Subsequently, an effective sampling principle based on the proposed measurement matrix is given as follows:

Suppose the original signal is $S=\left[s_{1}, s_{2}, \cdots s_{n}\right]$, when the terminal received $\mathrm{M}$ measurement $A_{M}=\left[a_{1}, a_{2}, \cdots, a_{M}\right]$ based on our measure scheme, the M-th recovery result is $S^{(M)}$. If the later results are all equivalent, that is $S^{(M+1)}=\cdots=S^{(M+\ell)}=S^{(M)}$, then $P\left\{S^{(M)}=S\right\} \geq 1-\sqrt{2}^{-\ell}$

Based on this principle, the sink could ensure whether sampling should be stopped with accurate reconstruction. In this case, the number of transmission in intra-class is $m \times|R|$, where $m$ is the number of measurement. Hence, the number of transmission in the whole network is

$$
m \times|R|+\sum_{r \in R}(|S(r)|-1)
$$

\section{Discussion}

This section discusses several important applicationrelated subjects, which are ratio of compression and rule of sampling for collaborative transmission.

\subsection{Ratio of Compression}

As Fig. 1 (a) shows, there are 20 sensors in the network and one of them is the sink sensor. Suppose the number of measurement is 7 , then the rest nineteen sensors must transmit data package 7 times according to the CS theory. The digits in parentheses denote the necessary number of transmitting based on conventional methods (called non-CS).
Obviously, the required amount of transmitting using CS is more than one using non-CS. To address this problem, a simple yet effective mixed idea is considered, which is illustrated in Fig. 1 (b). An essential difference is that each sensor which is close to the bottom only transmits its data to its upstream sensor using traditional methods until a certain sensor's children reaches to $7-1=6$. In this case, these branch sensors only need to transmit the same number with one in parentheses. Afterwards, CS is applied in remaining sensors, the number of whose children is equal to or greater than 6 . In this case, the relative ratio of compression between our scheme and natural CS is $48 / 133=36.09 \%$. It is clearly for the whole network that the number of transmitting decreases and energy cost reduces. Experiments are illustrated in Section V.

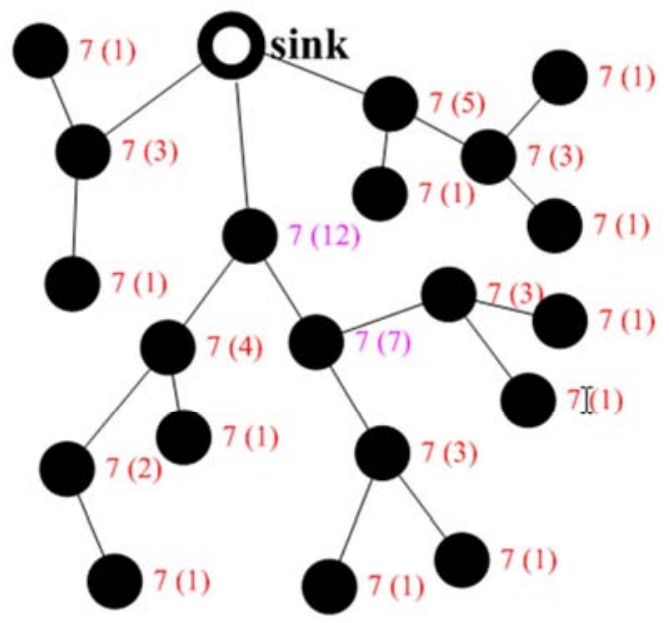

(a) Natural CS v. s. no-CS

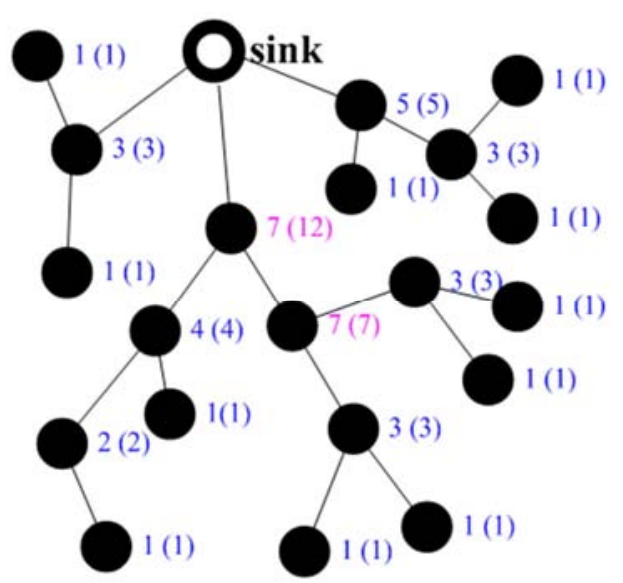

(b) Our scheme v. s. no-CS

Fig. 1. Comparison of the number of transmission.

\subsection{Rule of Sampling}

According the sampling principle, $S=S^{(M)}$ is high probability if $N^{2} \sqrt{2}^{1-M}$ is much less than 1 , since the elements of $a_{i}$ belong to finite set $\{-1,0,1\}$ and then a submatrix $A_{M}$ whose size is $\mathrm{M} \times \mathrm{M}$ can be singular with non-zero probability. 


\section{Experiments}

Sensors are used to gather temperature, illumination and magnetism of environments outdoors. After information coverage, data sensed by wake sensors and estimates of crucial sensors are transmitted to sink sensor MTS310. The protocol system of wireless network in our experiments includes three protocols of layers, which are physical layer, data link layer and net layer. The protocol of the former two is IEEE 802.15 .4 and the protocol of the last is XMesh that is a multi-hop, Ad-hoc, mesh network protocol.

To display the stability of the performance in the real applications, IRIS sensors in our experiments are divided into four groups, the numbers of which are 20,50,100 and 150 respectively. They are all connected in a self-organized way to gather temperature, illumination and magnetism 3 times per minute. To guarantee the accurate of the results, average values of the algorithms are calculated through 1500 times.

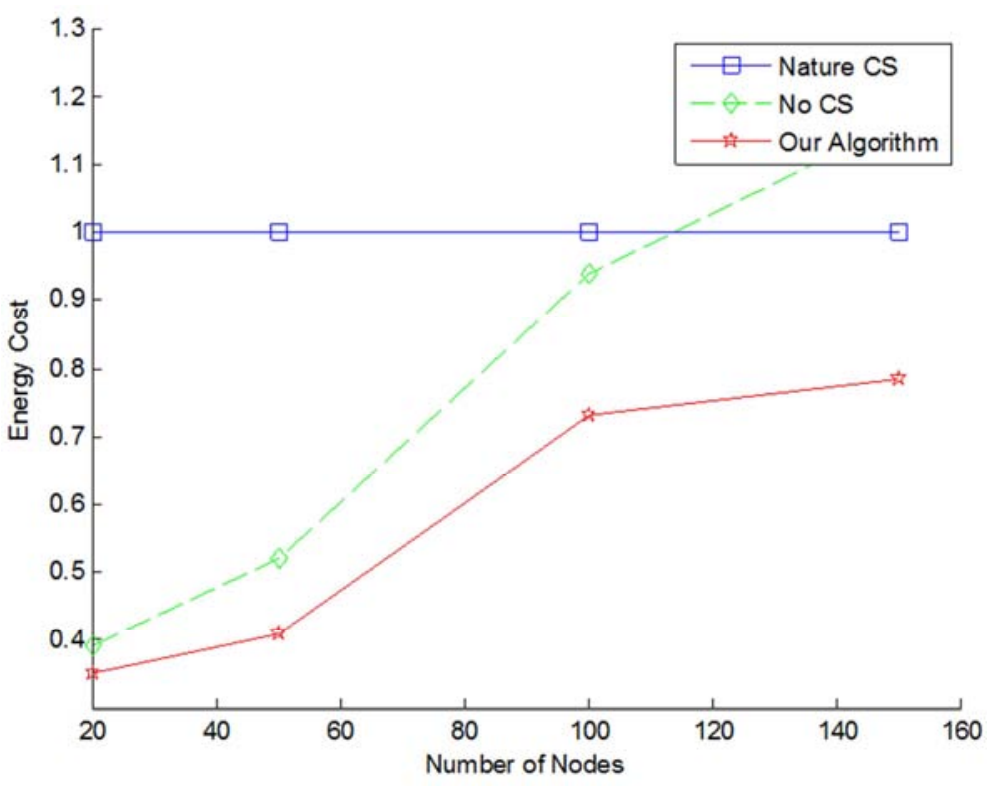

(a) the relation of energy cost and the number of nodes

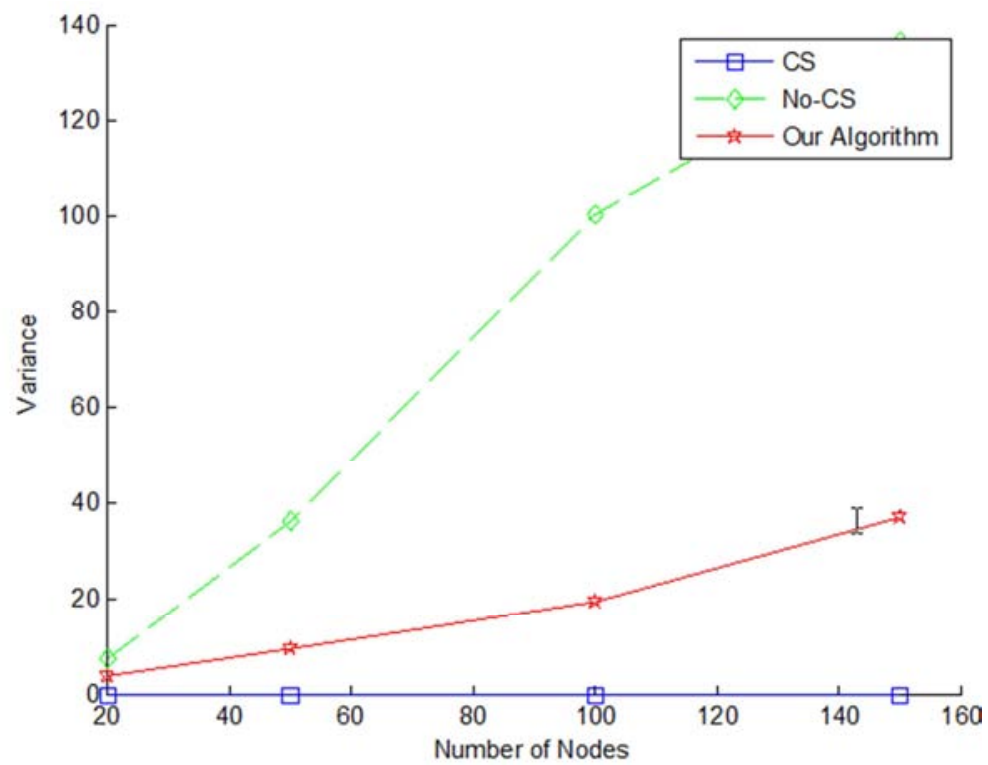

(b) the relation of variance and the number of nodes

Fig. 2. Our transmission mode V. S. Natural CS and No CS.

Firstly, we compare our transmission mode to natural CS and non-CS. According to the Fig. 2 (a), relative energy costs of three schemes are represented. Energy cost of natural CS is set to 1 which is illustrated by the straight line. The results show that our transmission mode costs less energy than others. Besides, both our scheme and non-CS are better than CS method, since the number of sensors in classes is less than the number of measurement. As the number of sensors increase, the advantages of CS are exploited and more energy is saved by our scheme. Fig. 2 (b) illustrates the state of 
balancing load. It is undoubted that the load of the network using CS is the optimal equilibrium; however, its energy cost is the most according to the analysis of Section II-A. In practice, our mixed scheme cost least energy as well as preserving the load balancing. Furthermore, the load of the network is stable as its scale enlarges, compared with more and more imbalanced situation using non-CS.

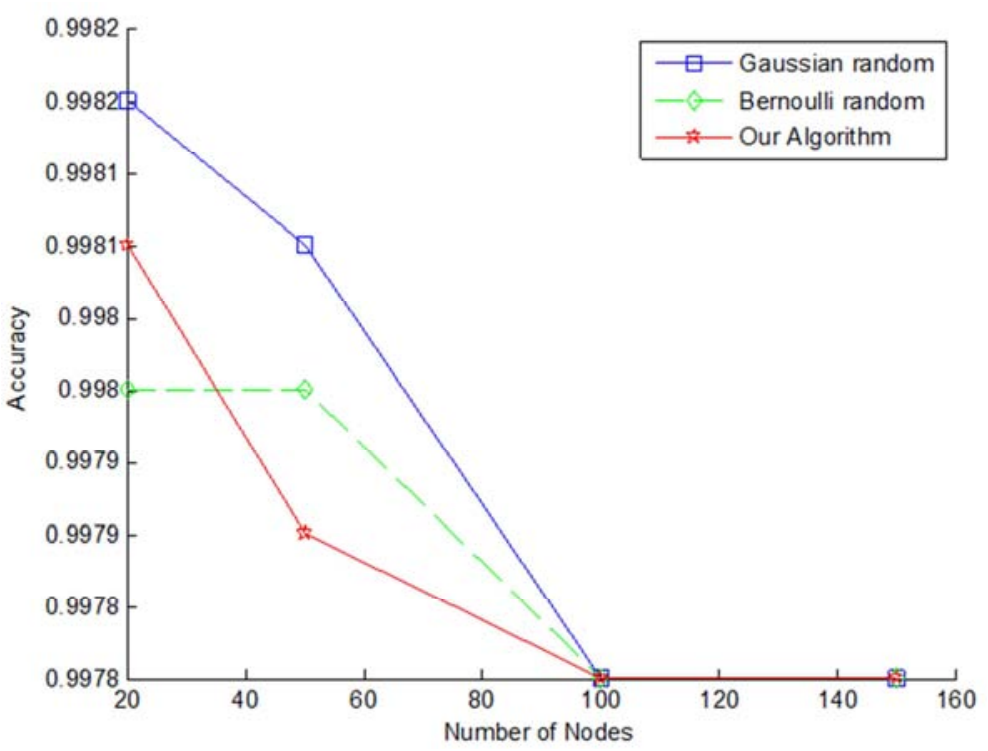

(a) our measurement and random measurement

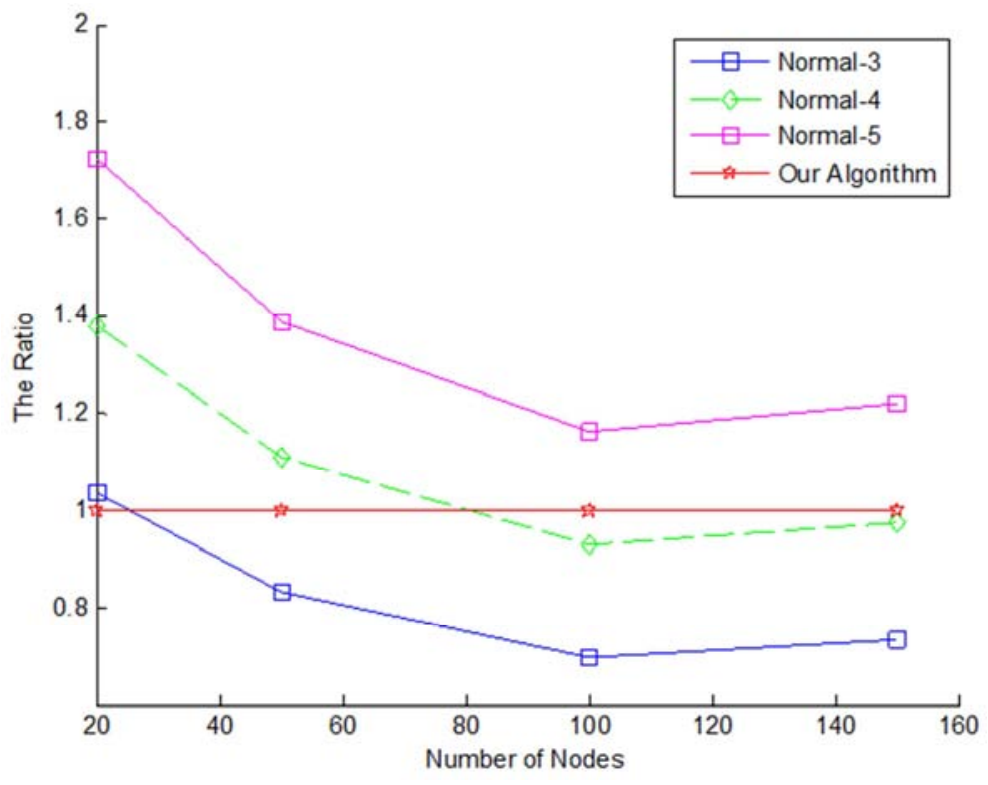

(b) our sampling and normal sampling

Fig. 3. Our measurement and sampling V.S. CS.

Furthermore, we compare our measurement matrix to random matrices, including Gaussian random and Bernoulli random. Fig. 3 (a) illustrates that performances of three measurement matrices are similar since our measurement matrix is as good as random matrices. Then the ratio of $n_{s p} / n_{\text {normal }}$ is demonstrated in Fig. 3 (b), where $n_{s p}$ denotes sampling numbers by using sampling principle and $n_{\text {normal }}$ denotes 3, 4, 5 times of sparsity of signals, which are normal sampling numbers based on the CS theory. The results reveal our sampling principle is effective and could reduce energy cost increasingly as the scale of the network growing. That is, it can be applied in a large-scale network.

\section{Conclusion}

In this paper, we propose a collaborative communication mechanism based on classification CS. In the case of collaborative transmission, an effective transmission method is proposed in which both non-CS and CS are adopted. In the process of transmission, all sensors in a class convey their data to the relay node of this class, and then the relay node 
produces a new data and transmits to the sink. After receiving data, a sampling principle is proposed to reduce redundant samplings, which gives the theoretical low bound of the accuracy of signal reconstruction. Experiments show that our transmission mechanism outperforms natural CS and routing scheme.

\section{References}

[1] J. Haldar, and D. Hernando, and ZP Liang, "Compressed Sensing in MRI with non-Fourier encoding", IEEE Trans. Med. Imag, vol. 30, pp. 893-903, 2013.

[2] J. Wu, and F. Liu, and LC Jiao, and X. Wang, "Compressive Sensing SAR Image Reconstruction Based on Bayesian Framework and Evolutionary Computation", IEEE Trans. Image Processing, no. 99, pp. 1-1, 2014.

[3] H. Yang, K. Tang, et al, "An adaptable CS-based transmission scheme validated on the real-world system," IEEE INFOCOM 2016

[4] Z. Charbiwala, S. Chakraborty, S. Zahedi, Y. Kim, M. B. Srivastava, T. He and C. Bisdikian. Compressive Oversampling for Robust Data Transmission in Sensor Networks. In INFOCOM, 2010.

[5] H. Yang, et al, "A Practical Information Coverage Approach in Wireless Sensor Network," Information Processing Letters, vol. 115, no. 1, pp. 6-10, 2015.

[6] C. Feng, W. S. A. Au, et al. Compressive sensing based positioning using RSS of WLAN access points. In INFOCOM, 2010.
[7] PS. C. Thejaswi, T. Tran, and J. Zhang. When compressive sampling meets multicast: Outage analysis and subblock network coding. In INFOCOM, 2011.

[8] A. Wani, etc. Compressive Sampling for Energy Efficient and Loss Resilient Camera Sensor Networks. In INFOCOM, 2011.

[9] Z. Li, Y. Zhu, H. Zhu, and M. Li. Compressive Sensing Approach to Urban Traffic Sensing. In ICDCS, 2014.

[10] H. Yang, et al, "Distributed Compressed Sensing in Wireless Local Area Networks," International Journal of Communication Systems, vol. 22, no. 11, pp. 2723-2743, 2014.

[11] H. Yang, L. Huang, H. Xu, "Distributed Compressed Sensing in Vehicular Ad-hoc Network," Ad hoc \& Sensor Wireless Networks, vol. 25, no. 1-2, pp. 121-145, 2015.

[12] E. J. Cand'es, J. Romberg, and T. Tao, "Robust Uncertainty Principles: Exact Signal Reconstruction from Highly Incomplete Frequency Information", IEEE Trans. Inform. Theory, vol. 52, no. 2, pp. 489-509, 2006.

[13] David L. Donoho, "Compressed sensing", IEEE Trans. on Inform. Theory, vol. 52, no. 4, pp. 1289-1306, 2006.

[14] E. Candes, "The Restricted Isometry Property and its Implications for Compressed Sensing", Comptes Rendus Mathematique, vol. 346, no. 9. pp. 589-592, 2008.

[15] M. F. Duarte, and S. Sarvotham, and D. Baron, and M. B. Wakin, and R. G. Baraniuk, "Distributed Compressed Sensing of Jointly Sparse Signals", Asilomar Conf. Signals, Sys., Comput, pp. 1537-1541, 2005. 